\title{
Impact of invasive fungal infection on outcomes of severe sepsis: a multicenter matched cohort study in critically ill surgical patients
}

\author{
Guo-Hao Xie ${ }^{1}$, Xiang-Ming Fang ${ }^{1}$, Qiang Fang ${ }^{1}$, Xin-Min Wu2 ${ }^{2}$, Yu-Hong Jinn', Jun-Lu Wang ${ }^{4}$, Qu- \\ Lian Guo ${ }^{5}$, Miao-Ning Gu${ }^{6}$, Qiu-Ping $X^{7}$, Dong-Xin Wang ${ }^{2}$, Shang-Long Yao ${ }^{8}$, Shi-Ying Yuan ${ }^{8}$, \\ Zhao-Hui Du ${ }^{9}$, Yun-Bo Sun ${ }^{10}$, Hai-Hong Wang ${ }^{7}$, Shui-Jing Wu ${ }^{1}$ and Bao-Li Cheng ${ }^{1}$
}

\author{
1Department of Anesthesiology and Intensive Care Unit, the First Affiliated Hospital, School of Medicine, Zhejiang University, 79 Qingchun Road, \\ 310003, Hangzhou, China \\ 2Department of Anesthesiology and Intensive Care Unit, the First Affiliated Hospital, School of Medicine, Peking University, 8 West Shenku Avenue, \\ 100034, Beijing, China \\ 3Intensive Care Unit, Lihuili Hospital, School of Medicine, Ningbo University, 57 Xingning Road, 315040, Ningbo, China \\ 4Department of Anesthesiology, the First Affiliated Hospital, Wenzhou Medical College, 2 Fuxue Road, 325000, Wenzhou, China \\ ${ }^{5}$ Department of Anesthesiology and Intensive Care Unit, Xiangya Hospital, Xiangya Medical College, Central South University, 87 Xiangya Road, \\ 410008, Changsha, China \\ ${ }^{6}$ Department of Anesthesiology and Intensive Care Unit, South Hospital, South Medical University, 1836 Guangzhou Road, 510515, Guangzhou, \\ China \\ ${ }^{7}$ Department of Anesthesiology and Intensive Care Unit, Sir Run Run Shaw Hospital, School of Medicine, Zhejiang University, 3 Qingchun Road, \\ 310016, Hangzhou, China \\ ${ }^{8}$ Department of Anesthesiology and Intensive Care Unit, Union Hospital, Tongji Medicine College, Huazhong University of Science and Technology, \\ 1095 Jiefang Road, 430030, Wuhan, China \\ 9Intensive Care Unit, Zhongnan Hospital, School of Medicine, Wuhan University, 169 Donghu Road, 430071, Wuhan, China \\ ${ }^{10}$ Department of Anesthesiology and Intensive Care Unit, Qingdao University Hospital, School of Medicine, Qingdao University, 16 Jiangsu Road, \\ 266011, Qingdao, China
}

Corresponding author: Xiang-Ming Fang, xiangming_fang@163.com

Received: 13 Nov 2007 Revisions requested: 15 Dec 2007 Revisions received: 3 Jan 2008 Accepted: 16 Jan 2008 Published: 16 Jan 2008

Critical Care 2008, 12:R5 (doi:10.1186/cc6766)

This article is online at: http://ccforum.com/content/12/1/R5

(c) 2008 Xie et al.; licensee BioMed Central Ltd.

This is an open access article distributed under the terms of the Creative Commons Attribution License (http://creativecommons.org/licenses/by/2.0), which permits unrestricted use, distribution, and reproduction in any medium, provided the original work is properly cited.

\begin{abstract}
Introduction Fungal infection is increasingly common in critical illness with severe sepsis, but the influence of invasive fungal infection (IFI) on severe sepsis is not well understood. The aim of this study was to investigate the impact that IFI has on the outcomes of critically ill surgical patients with severe sepsis in China by means of matched cohort analysis; we also evaluated the epidemiologic characteristics of IFI in this population.
\end{abstract}

Methods Records for all admissions to 10 university hospital surgical intensive care units (ICUs) from December 2004 to November 2005 were reviewed. Patients who met criteria for severe sepsis were included. IFI was identified using established criteria based on microbiologic or histological evidence. A matched cohort study was conducted to analyze the relationship between IFI and outcomes of severe sepsis.
Results A total of 318 patients with severe sepsis were enrolled during the study period, of whom 90 (28.3\%) were identified as having IFI. A total of 100 strains of fungi (58\% Candida albicans) were isolated from these patients. Independent risk factors for IFI in patients with severe sepsis included mechanical ventilation ( $>3$ days), Acute Physiology and Chronic Health Evaluation score, coexisting infection with both Gram-positive and Gram-negative bacteria, and urethral catheterization $(>3$ days). Compared with the control cohort, IFI was associated with increased hospital mortality $(P<0.001)$, high hospital costs $(P=0.038)$, and prolonged stay in the ICU $(P<0.001)$ and hospital $(P=0.020)$.

Conclusion IFI is frequent in patients with severe sepsis in surgical ICUs and is associated with excess risk for hospital mortality, longer ICU and hospital stays, and greater consumption of medical resources. 


\section{Introduction}

Invasive fungal infection (IFI) is a severe clinical complication in immunocompromised patients, such as neutropenic patients, recipients of bone marrow or solid organ transplants, cancer patients receiving chemotherapy, and HIV-infected patients. However, during the past two decades, with advances in diagnostic and therapeutic interventions, critically ill patients with lesser degrees of immunocompromise, especially those in surgical and neonatal intensive care units (ICUs), have emerged as another population at high risk for IFI [1-3].

Sepsis is the body's systemic inflammatory response to infection. It is considered severe when it is associated with acute organ dysfunction. According to epidemiologic studies [4-13], severe sepsis has become a leading cause of morbidity and mortality in critical illness, and etiologic evidence [14] indicates that the incidence of fungal infection in septic patients is increasing. Fungal organisms are common pathogens in surgical patients and patients suffering from severe sepsis. Raymond and coworkers [15] observed that fungal infection accounted for $12.3 \%$ of all episodes of surgical infection in a study conducted over 38 months. Finfer and colleagues [8] reported that fungi accounted for approximately $12.1 \%$ of all microbial isolates in their study of severe sepsis among adult admissions to ICUs in Australia and New Zealand [8]. Recently, the large pan-European Sepsis Occurrence in Acutely III Patients (SOAP) study [4] reported that fungal infection was observed in 17\% of all septic patients in European ICUs. Furthermore, IFI was found to be associated with excess mortality, hospital stay, and cost $[16,17]$. Annane and coworkers [18] identified fungal infection as an independent risk factor for increased mortality among patients with septic shock. However, fungal infection was not found to be among the significant predictors of fetal outcome in some other studies [4].

In the present study we attempted to determine the impact that IFI has on outcomes of severe septic patients in multiple surgical ICUs in China. We undertook a matched cohort study in which severe septic patients with and without IFI were matched for unit, age, sex and severity of illness.

\section{Materials and methods}

\section{Study population and data collection}

The present study was conducted in the surgical ICUs of 10 university hospitals in six major provinces from 1 December 2004 to 30 November 2005 (participating centers are listed under Acknowledgement [see below]). The study protocol was approved by the ethical committee in each participating center, and informed consent was waived because of the observational nature of this study. All adult patients (age $\geq 18$ years) admitted to the participating surgical ICUs during the period of observation and who met criteria for severe sepsis were included in the study cohort. These patients were evalu- ated prospectively by investigators daily by chart review and interview of ICU physicians. For all enrolled patients, the following data were collected: age, sex, primary diagnosis, chronic comorbidities, clinical data needed for calculation of Acute Physiology and Chronic Health Evaluation (APACHE) II score during the first 24 hours after ICU admission [19], daily Sequential Organ Failure Assessment (SOFA) [20,21], microbiological and clinical infection data, antibiotics and antifungal agents administered, hospital costs, and hospital outcome. The chronic comorbidity system in this study was constructed by selecting ICD-9-CM codes suggestive of chronic disease within separate organ systems. 'Cost' was defined as the total expenditures on medical care attributable to a patient in the hospital, including charges for medical care, nursing, medication, and laboratory testing. Indirect expenses were not calculated.

Those who were readmitted and had been included on their first admission were not included for a second time. If one patient suffered more than one episode of IFI, then only the first episode was included in the study.

For the matched cohort study, patients with severe sepsis suffering IFI during their ICU stay were matched to control patients with severe sepsis but without IFI. We attempted to match each individual with severe sepsis with a control patient in the same center. The matching process included the following factors: sex, age ( \pm 10 years) and APACHE II score $( \pm 3)$ [22]. If one patient could be matched to two or more control patients, then the control patient with the closest APACHE II score was selected.

\section{Definitions}

The criteria for IFI were defined based on the study conducted by and coworkers [23]. This definition requires the presence of fungemia, specifically blood culture yielding fungi in patients with temporally related clinical signs and symptoms compatible with relevant organism. It also requires IFI to be present in other sites, to be confirmed histopathologically or cytopathologically in needle aspirate or biopsy specimen, or fulfilling the following four criteria: positive culture result for samples obtained via sterile procedure from normally sterile sites, excluding urine and mucous membranes; compatible clinical and radiologic manifestations; no evidence of infection caused by micro-organisms other than fungus; and improvement in signs and symptoms, and radiology after use of antifungal agents. Treated as a special case, invasive pulmonary aspergillosis was diagnosed only if there was positive histologic testing and/or culture after biopsy or autopsy, as defined by Vandewoude and coworkers [24]. Detection of fungi and speciation of isolates were performed at the laboratories of participating hospitals, in accordance with their standard protocols. The antifungal activity of amphotericin B, fluconazole and itraconazle was also tested in these laboratories. The time 
of onset of IFI was defined as the date in which the first positive culture or biopsy was identified.

Sepsis was defined in accordance with the American College of Chest Physicians/Society of Critical Care Medicine consensus conference definition [25]. Severe sepsis was defined as sepsis plus sepsis-induced acute organ dysfunction (occurring in at least one organ), as indicated by a SOFA score of 2 or more for the organ in question.

'Combination antibiotic therapy' in this report refers to antibiotic therapy involving more than one of the following types of antibiotic, administered daily for at least 3 days: penicillins, cephalosporins, carbapenem, macrolides, glycopeptides, aminoglycosides, quinolones, sulfonamides, and others.

\section{Statistical analyses}

Continuous data are presented as median with interquartile range (25th to 75th percentiles) and compared using MannWhitney test, except for the variable cost, which is presented as mean \pm standard and compared using the $t$ test. Categorical data are shown as percentages and compared using the $\chi^{2}$ test. In the matched cohort study, comparisons of paired baseline characteristics were performed using the paired Student's $t$-test and the McNemar test for continuous and categorical variables, respectively. The matched risk ratio and excess risk were expressed by ratio and difference in mortality rates between exposed and unexposed patients, respectively. The tests were two-sided, and $P<0.05$ wa deemed to reflect statistical significance.

A multivariate nonconditional logistic regression analysis was conducted in patients with severe sepsis, with occurrence of IFI in the ICU as a dependent factor. Variables considered in multivariate modeling included age, sex, chronic comorbidity, coexisting bacterial infection, APACHE II score, mechanical ventilation ( $>3$ days), central venous catheterization ( $>3$ days), urethral catheterization ( $>3$ days), and combination antibiotic therapy in the ICU (for patients with severe sepsis and IFI, only the data before the onset of IFI were adopted in the regression). Forward elimination, which employed a combination of the procedures used in the forward entry and backward removal methods, was adopted in the regression analysis. The effect on occurrence of IFI in patients with severe sepsis was considered statistically significant if the regression coefficient associated with IFI was at a level of $P<0.05$. Statistical analysis was conducted using SPSS 13.0 for Windows (SPSS Inc., Chicago, IL, USA).

\section{Results \\ Incidence and demographics}

From 1 December 2004 to 30 November 2005, 318 patients were identified as having severe sepsis and hence were enrolled in the study cohort, including 206 men (64.8\%) and 112 women (35.2\%). Among these 318 patients, 90 (28.3\%)
Table 2

\begin{tabular}{ll}
\hline Characteristics of fungal infection & \\
\hline Characteristics & Episodes $(n[\%])$ \\
\hline Pathogens & \\
\hline Candida albicans & $58(58.0)$ \\
Candida tropicalis & $17(17.0)$ \\
Candida glabrata & $15(15.0)$ \\
Candida parapsilosis & $3(3.0)$ \\
Aspergillus & $3(3.0)$ \\
Others or unclassified & $4(4.0)$ \\
Site of infection & \\
Lung & $62(56.4)$ \\
Abdomen & $25(22.7)$ \\
Bloodstream or catheter related & $15(13.6)$ \\
Other sites & $8(7.3)$ \\
\end{tabular}

developed IFI in the ICU, including 20 (6.3\%) suffering from fungemia. A comparison of demographic characteristics for the 90 patients with IFI versus those of patients without IFI (control patients) is presented in Table 1.

\section{Characteristics of fungal infection}

In all 90 patients with IFI, 100 episodes of invasive fungal infection were identified, including 58 involving Candida albicans $(58.0 \%), 17$ C. tropicalis $(17.0 \%), 15$ C. glabrata (15.0\%), three C. parapsilosis (3.0\%), three Aspergillus spp. (3.0\%), and four other species or unclassified (4.0\%). Eight patients were infected with two or more fungal species (Table 2). Resistance to fluconazole was detected in 24 isolates.

Lung was the most common site of IFI, followed by abdominal, and bloodstream or catheter-related infection (Table 2). Twenty patients suffered from multi-site infection.

\section{Interventions}

In all, $78.9 \%, 82.2 \%$, and $93.3 \%$ of the 90 patients with IFI underwent mechanical ventilation, central venous catheterization, and urethral catheterization, respectively. However, patients with severe sepsis but without IFI were likely to undergo fewer invasive procedures tosse patients with IFI (Table 1).

Combination antibiotic therapies were adminstered to 45 (50.0\%) of the 90 patients before the onset of IFI, which was not statistically significant from that in patients with severe sepsis but without IFI during the entire stay in the ICU $(57.0 \%$; $P=0.263)$. In the participating surgical ICUs, antifungal agents were chosen based on the results of antifungal susceptibility testing. Fluconazole was administered to 66 
Table 1

Demographic characteristics of severe sepsis patients with and without invasive fungal infection

\begin{tabular}{|c|c|c|c|}
\hline Variables & $\begin{array}{l}\text { Severe sepsis patients with IFI } \\
(n=90)\end{array}$ & $\begin{array}{l}\text { Severe sepsis patients without IFI } \\
(n=228)\end{array}$ & $P$ value \\
\hline \multicolumn{4}{|l|}{ Baseline descriptors } \\
\hline Age (years) & $65(50-76.25)$ & $61.5(45-73)$ & NS \\
\hline Sex (male; $n[\%])$ & $57(63.3 \%)$ & $149(65.4 \%)$ & NS \\
\hline Admission APACHE II score (mean [IQR]) & $21(17-27)$ & $18(13-23)$ & 0.001 \\
\hline Admission SOFA score(mean [IQR]) & $8(6-12)$ & $8(5-11.75)$ & NS \\
\hline Comorbidity $(n[\%])$ & $71(78.9 \%)$ & $165(72.4 \%)$ & NS \\
\hline Cancer $(n[\%])$ & $14(15.6 \%)$ & $24(10.5 \%)$ & NS \\
\hline Diabetes mellitus ( $n[\%])$ & $15(16.7 \%)$ & $26(11.4 \%)$ & NS \\
\hline \multicolumn{4}{|l|}{ Interventions } \\
\hline Mechanical ventilation $(n[\%])$ & $71(78.9 \%)$ & $75(32.9 \%)$ & $<0.001$ \\
\hline Central venous catheterization ( $n[\%])$ & $74(82.2 \%)$ & $122(53.5 \%)$ & $<0.001$ \\
\hline Urinary catheterization ( $n[\%])$ & $84(93.3 \%)$ & $137(60.1 \%)$ & $<0.001$ \\
\hline Arterial catheterization ( $n[\%])$ & $51(56.7 \%)$ & $112(49.1 \%)$ & NS \\
\hline Total parenteral nutrition $(n[\%])$ & $49(54.4 \%)$ & $99(43.4 \%)$ & NS \\
\hline Corticosteroids or immunodepressant drugs ( $n[\%]$ ) & $22(24.4 \%)$ & $45(19.7 \%)$ & NS \\
\hline Renal replacement therapy $(n[\%])$ & $21(23.3 \%)$ & $28(12.3 \%)$ & 0.024 \\
\hline \multicolumn{4}{|l|}{ Outcomes } \\
\hline ICU LOS (days; mean [IQR]) & $16(7.75-31)$ & $5(2-10)$ & $<0.001$ \\
\hline Hospital LOS (days; mean [IQR]) & $29.5(17.75-50.25)$ & $19(10.25-36)$ & $<0.001$ \\
\hline Daily costs $(\$ ;$ mean $\pm \mathrm{SD})$ & $520 \pm 319$ & $481 \pm 437$ & NS \\
\hline Hospital costs $(\$ ;$ mean \pm SD) & $17,051 \pm 14,183$ & $8,474 \pm 9,484$ & 0.001 \\
\hline Hospital mortality & $61(67.8 \%)$ & $94(41.2 \%)$ & $<0.001$ \\
\hline
\end{tabular}

APACHE, Acute Physiology and Chronic Health Evaluation; ICU, intensive care unit; IQR, interquartile range; IFI, invasive fungal infection; LOS, length of stay; NS, not significant; SD, standard deviation; SOFA, Sequential Organ Failure Assessment.

patients with IFI, whereas itraconazole was administered to 22 patients and amphotericin B to two patients. Also, 75 patients received intravenous antifungal agents.

\section{Predictors of invasive fungal infection}

The variables retained in the final model in the multivariate logistic regression and hence associated with increased risk for IFI in patients with severe sepsis included the following: mechanical ventilation ( $>3$ days), APACHE II score, coexisting infection with both Gram-positive and Gram-negative bacteria, and urethral catheterization ( $>3$ days; Table 3 ).

\section{Mortality}

The observed hospital mortality rate was significantly higher in patients with severe sepsis and IFI than in those patients without IFI (67.8\% versus $41.2 \% ; P<0.001)$. There was no significant difference in hospital mortality between male and female patients with IFI (61.4\% versus $51.5 \% ; P=0.384)$.

\section{Resource use and costs}

The median hospital and ICU lengths of stay (LOSs) for all 318 patients with severe sepsis were 7 (3 to 14) days and 22 (12 to 39) days, respectively. Patients with severe sepsis and IFI had longer ICU and hospital LOSs than did those without IFI (ICU LOS: 16 [7.75 to 31] days versus 5 [2 to 10] days, $P<$ 0.001 ; hospital LOS: 29.5 [17.75 to 50.25] days versus 19 [10.25 to 36.00] days, $P<0.001)$. Among patients with severe sepsis and IFI, the ICU and hospital LOSs for men were comparable to those for women (ICU LOS: 18 [8 to 30] days versus 13 [7 to 32] days, $P=0.505$; hospital LOS: 28 [17 to 56 ] days versus 31 [20.5 to 47.5 ] days, $P=0.657$ ).

The mean hospital cost was much higher in severe septic patients with IFI than those without IFI $(\$ 17,051 \pm 14,183$ versus $\$ 8,474 \pm 9,484, P=0.001)$. However, the mean daily costs were similar between the two groups $(\$ 520 \pm 319$ versus $\$ 481 \pm 437, P=0.59$ ). 
Table 3

Logistic regression analysis in patients with severe sepsis, with occurrence of IFI in the ICU as the dependent factor

\begin{tabular}{lll}
\hline Variable & OR $(95 \% \mathrm{Cl})$ & $P$ value \\
\hline Mechanical ventilation $(>3$ days $)$ & $3.28(1.67-6.45)$ & 0.001 \\
APACHE II score & $1.43(1.17-1.76)$ & 0.001 \\
$\begin{array}{l}\text { Coexisting infection by both Gram- } \\
\text { positive and Gram-negative bacteria }\end{array}$ & $4.06(2.23-7.38)$ & $<0.001$ \\
Urethral catheterization $(>3$ days $)$ & $3.97(1.45-10.89)$ & 0.007 \\
\hline
\end{tabular}

A total of 318 patients were included in this analysis. APACHE, Acute Physiology and Chronic Health Evaluation; APS, Acute Physiology Score; $\mathrm{Cl}$, confidence interval; OR, odds ratio.

\section{Results of matched cohort study}

Sixty patients with severe sepsis and IFI could be matched to 60 patients with severe sepsis but without IFI (matched control patients), based on unit, sex, age, and APACHE II score. The patients for whom matched control patients could be identified accounted for $66.7 \%$ of the 90 patients with severe sepsis and IFI, and these matched 60 patients with severe sepsis and IFI had similar mortality to that in the unmatched 30 patients (Table 4). Compared with control patients, markedly higher hospital mortality, ICU LOS, hospital LOS and hospital costs, and more aggressive interventions (mechanical ventilation, central venous catheterization, and urinary catheterization) were observed in matched patients with severe sepsis and IFI. However, daily costs were similar between the two groups (Table 5). IFI was associated with a matched excess risk for death in hospital of $20 \%(70.0 \%$ versus $50.0 \%, P=$ $0.023)$, and the matched risk ratio was 1.4 .

\section{Discussion}

The present study focused on IFI in patients with severe sepsis in surgical ICUs, and it contributes important additional information to that from the large studies on epidemiology of severe sepsis published thus far. The study identified a $28.3 \%$ incidence of IFI in critically ill patients with severe sepsis. Lung and abdomen were the most common sites of IFI. C. albicans, C. tropicalis, and C. glabrata were the predominant species and comprised $90 \%$ of all isolated strains. Mechanical ventilation ( $>3$ days), APACHE II score, infection with both Grampositive and Gram-negative bacteria, and urinary catheterization ( $>3$ days) were identified as independent risk factors for IFI in patients with severe sepsis. Compared with the control patients with severe sepsis but without IFI, the matched patients with severe sepsis and IFI had higher hospital mortality, ICU LOS, hospital LOS and hospital costs, and received more aggressive interventions.

We found a high incidence of IFI (28.3\%) in critically ill surgical patients suffering from severe sepsis. This is higher than that reported in the SOAP study [4], which identified fungal infection in approximately $17 \%$ of the critically ill septic patients in several European countries. This may be because the cohort we studied was more susceptible to fungal infection, as factors that may compromise the integrity of gastrointestinal mucosa and facilitate fungal translocation (such as poor nutrition, trauma, hypotension and therapy with steroids, as well as ischemia and reperfusion) are common in the ICU population. Moreover, injury, trauma, and blood loss in surgical patients, which result in marked depression in cell-mediated immunity, may specifically be associated with high incidence of IFI [26]. However, some factors other than underlying disease and characteristics of patients admitted to the ICU may also contribute to the high incidence. First, in accordance with the criteria used in this study, some of the patients were diagnosed as having IFI but without biopsy, which may lead to enrollment of patients with fungal colonization and overestimation of IFI in surgical patients with severe sepsis. Secondly, most of the enrolled patients with severe sepsis had been hospitalized in the surgical wards before their ICU admission and were routinely administered prophylactic antibiotic treatment; hence, they were more susceptible to nosocomial fungal infection. Third, lung is the most commonly impaired organ in severe sepsis, and mechanical ventilation is instituted in most patients suffering from severe sepsis. Consequently, these individuals are prone to pulmonary fungal infection, because endotracheal tubes may facilitate the intrusion of endogenous or exogenous fungal organisms. In the present study, lung was the major site of fungal infection, and $82.3 \%$ of IFI patients who suffered from pulmonary fungal infection received mechanical ventilation for at least 3 days. Finally, the higher occurrence of IFI may also reflect a horizontal transmission of fungal infection in the ICU, because a previous study [27] found Candida spp. to be present on the hands of $39 \%$ of surgical ICU staff.

Lack of DNA analysis of fungal isolates prevented interpretation, but our findings are in accordance with those of a previous study [28] that identified a $23.96 \%$ incidence of fungal infection among patients admitted to an ICU in a Chinese university hospital. Therefore, infection control in ICUs within China may require improvement and standardization. More attention should be given to sanitary precautions in ICU in order to prevent fungal infection; such precautions include using high-efficiency particulate air filters, changing the breathing circuits of ventilators periodically, and applying endotracheal tubes with a dorsal lumen to allow drainage of respiratory secretions. Standard protocols should be established to evaluate the appropriateness of administered aggressive interventions.

The spectrum of fungal species identified in this study was consistent with that in previous studies [29-31], which found that C. albicans and non-albican species accounted for approximately half of reported cases of IFI. We found four factors to be independently associated with risk for IFI in severe sepsis (mechanical ventilation $>3$ days], APACHE II score, infection with both Gram-positive and Gram-negative bacteria, 
Critical Care Vol 12 No 1 Xie et al.

Table 4

Comparison between matched and unmatched severe septic patients with IFI

\begin{tabular}{|c|c|c|c|}
\hline Variables & Matched patients with IFI $(n=60)$ & Unmatched patients with IFI $(n=30)$ & $P$ value \\
\hline Age (years; mean [IQR]) & $67.5(52.25-75.75)$ & $62(45.5-79.5)$ & NS \\
\hline Sex (male; \%) & $71.7 \%$ & $46.7 \%$ & 0.019 \\
\hline Admission APACHE score (mean [IQR]) & $20(16.25-25)$ & $25(17-32)$ & 0.035 \\
\hline Admission SOFA score (mean [IQR]) & $8(6-10.75)$ & $9(7-14)$ & NS \\
\hline ICU LOS (days; mean [IQR]) & $17.5(10-31)$ & $13(4.5-30.5)$ & NS \\
\hline Hospital LOS (days; mean [IQR]) & $30(19.25-50)$ & $29(16-55.5)$ & NS \\
\hline Daily costs $(\$ ;$ mean $\pm S D)$ & $526 \pm 319$ & $501 \pm 332$ & NS \\
\hline Hospital costs ( $\$$; mean \pm SD) & $16,804 \pm 14,102$ & $17,749 \pm 15,081$ & NS \\
\hline hospital mortality (\%) & $70.0 \%$ & $63.3 \%$ & NS \\
\hline
\end{tabular}

APACHE, Acute Physiology and Chronic Health Evaluation; ICU, intensive care unit; IFI, invasive fungal infection; LOS, length of stay; NS, not significant; SD, standard deviation; SOFA, Sequential Organ Failure Assessment.

Table 5

Comparison between severe septic patients with and without IFI in the matched cohort study

\begin{tabular}{|c|c|c|c|}
\hline Variable & $\begin{array}{l}\text { Severe sepsis patients with IFI } \\
(n=60)\end{array}$ & $\begin{array}{l}\text { Severe sepsis patients without IFI } \\
(n=60)\end{array}$ & $P$ value \\
\hline \multicolumn{4}{|l|}{ Baseline descriptors } \\
\hline Age (years; mean [IQR]) & $67.5(52.25-75.75)$ & $68(54-74)$ & NS \\
\hline Admission APACHE II score (mean [IQR]) & $20(16.25-25)$ & $20(16.25-23.75)$ & NS \\
\hline Admission SOFA score (mean [IQR]) & $8(6-10.75)$ & $8(5-12)$ & NS \\
\hline Co-morbidity ( $n[\%])$ & $47(78.3 \%)$ & $48(80.0 \%)$ & NS \\
\hline Cancer $(n[\%])$ & $9(15.0 \%)$ & $11(18.3 \%)$ & NS \\
\hline Diabetes mellitus ( $n[\%]$ ) & $10(16.7 \%)$ & $9(15.0 \%)$ & NS \\
\hline \multicolumn{4}{|l|}{ Interventions } \\
\hline Mechanical ventilation $(n[\%])$ & $49(81.7 \%)$ & $24(40.0 \%)$ & $<0.001$ \\
\hline Central venous catheterization ( $n[\%]$ ) & $51(85.0 \%)$ & $35(58.3 \%)$ & $<0.001$ \\
\hline Urinary catheterization ( $n[\%])$ & $58(96.7 \%)$ & $36(60.0 \%)$ & $<0.001$ \\
\hline Arterial catheterization ( $n[\%])$ & $41(68.3 \%)$ & $35(58.3 \%)$ & NS \\
\hline Total parenteral nutrition $(n[\%])$ & $35(58.3 \%)$ & $23(38.3 \%)$ & 0.036 \\
\hline Corticosteroids or immunodepressant drugs ( $n[\%]$ ) & $13(21.7 \%)$ & $16(26.7 \%)$ & NS \\
\hline Renal replacement therapy ( $n[\%])$ & $12(20.0 \%)$ & $11(18.3 \%)$ & NS \\
\hline \multicolumn{4}{|l|}{ Outcomes } \\
\hline ICU LOS (days; mean [IOR]) & $17.5(10-31)$ & $6.5(3-10)$ & $<0.001$ \\
\hline Hospital LOS (days; mean [IQR]) & $30(19.25-50)$ & $20(10.25-38.5)$ & 0.020 \\
\hline Mean daily costs $(\$ ;$ mean \pm SD) & $592 \pm 326$ & $469 \pm 396$ & NS \\
\hline Hospital costs $(\$ ;$ mean \pm SD) & $17,951 \pm 15,470$ & $10,023 \pm 12,347$ & 0.038 \\
\hline Hospital mortality ( $n[\%])$ & $42(70.0 \%)$ & $42(50.0 \%)$ & 0.023 \\
\hline
\end{tabular}

APACHE, Acute Physiology and Chronic Health Evaluation; ICU, intensive care unit; IQR, interquartile range; IFI, invasive fungal infection; LOS, length of stay; NS, not significant; SD, standard deviation; SOFA, Sequential Organ Failure Assessment. 


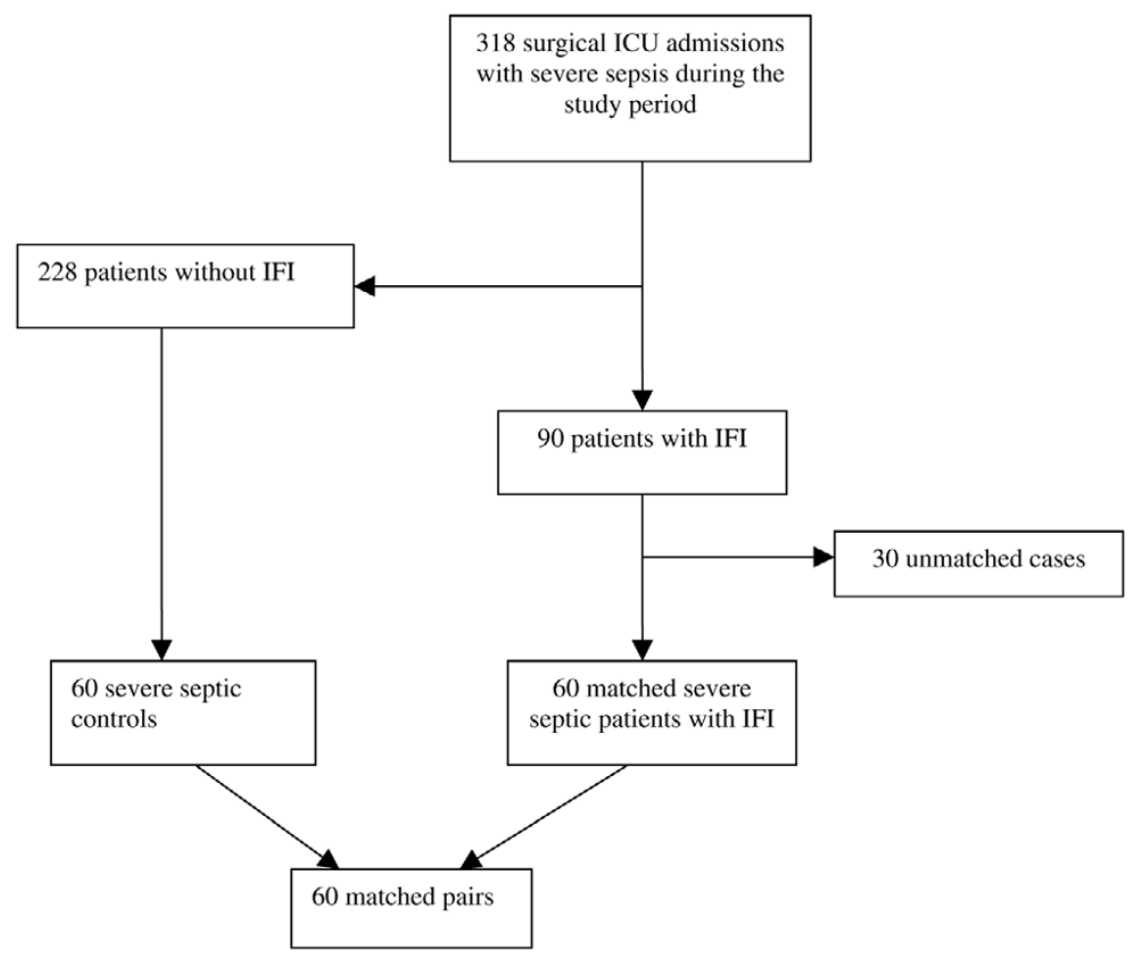

Flow diagram of the enrolled patients. Of the 318 enrolled patients with severe sepsis, 90 were identified as patients with invasive fungal infection (IFI), 60 of whom were 1:1 matched to control patients with severe sepsis but without IFI for unit, sex, age ( \pm 10 years) and Acute Physiology and Chronic Health Evaluation II score ( \pm 3 points). ICU, intensive care unit.

and urinary catheterization [ $>3$ days]). This is congruent with the findings of previous studies focusing on fungal infection in critical illness [32-34], except for coexisting infection with both Gram-positive and Gram-negative bacteria. The latter may be associated with greater severity of infection or use of broadspectrum antibiotics. However, logistic regression did not identify combination antibiotic therapy as an independent factor influencing the occurrence of IFI in this study. In the present study, combination antibiotic therapy was administered to $50 \%$ of the patients with IFI before the onset of IFI, which was similar to the proportion in patients without IFI during their ICU stay $(57 \%, P=0.263)$.

In order to minimize the influence of confounding factors in our evaluation of the relationship between IFI and outcome, a matched cohort study was conducted. Among the 90 patients with IFI, the 60 patients who could be matched to a control patient were similar to the 30 unmatched patients in terms of age, hospital LOS, ICU LOS, and hospital mortality, which indicates that these matched patients are representative of the whole group of IFI patients. Compared with the control patients with severe sepsis, the matched patients with severe sepsis and IFI had significantly greater hospital mortality. Therefore, this matched cohort study allowed rational estimation that IFI in patients with severe sepsis in surgical ICUs is associated with an excess risk for hospital death of $20 \%$, which is close to that reported in previous correlative studies [35-37]. Patients with IFI also had longer ICU and hospital LOSs than did control patients. Although the present study showed that IFI did not contribute to excess daily costs, it did correlate with excess hospital costs and consumption of medical resources as a result of the prolonged ICU and hospital LOSs.

A major advantage of our study is that a matched cohort study was conducted, and hence the fungus-related mortality was apparent. However, there are several limitations of this study. First, patients diagnosed as having IFI but without biopsy conformation were enrolled, in accordance with the criteria used, which may to a certain degree cause over-diagnosis of IFI. However, there is no consensus definition on IFI in non-neutropenic critically ill patients. Because of hemodynamic and/or respiratory insufficiency and coagulopathy of critical illness, and refusal by most of the Chinese families to allow antemortem or postmortem biopsy, it was rather difficult to diagnose IFI by positive culture from normally sterile sites, especially lower respiratory tract. Exclusion of all patients without biopsy conformation would inevitably have resulted in severe underestimation of IFI in the study population and altered the spectrum of fungal species identified. Therefore, we employed criteria to diagnose IFI patients without biopsy confirmation partly based on the findings of previous studies. Although our 
criteria may lead to over-diagnosis IFI in the study cohort, the findings based on it may be more objective. Second, this was a retrospective cohort study, although the database was constructed prospectively. Third, selection of control patients was mainly based on severity of illness at admission, as indicated by APACHE II score, but the severity of illness might have drifted apart between case patients and control patients since before the onset of IFI [38]. However, Blot and coworkers [39] found that expected mortality estimated from characteristics obtained on the first day of ICU admission correlated well with observed mortality in candidemic patients. Finally, this study included a relatively small sample to define the characteristics of IFI in a cohort with severe sepsis, in the most populous country in the world. However, there are no national hospital databases of sepsis available on the internet in today's China, and the financial and personnel resources supporting the present study were limited, both of which prevented our team from extending the study to more medical centers on a greater geographic scale.

\section{Conclusion}

The present study found IFI to be frequent in patients with severe sepsis in surgical ICUs, and to be associated with excess risk for death in hospital and greater consumption of medical resources. Therefore, in the process of diagnosing and treating severe sepsis in the ICU, attention should be given to the identification of patients who are at high risk for IFI, as well as prevention of and early intervention in IFI in these patients.

Key messages
Ninety severe septic patients (28.3\%) were identified
as patients with IFI.
- Mechanical ventilation ( $>3$ days), APACHE II score,
coexisting infection with both Gram-positive and Gram-
negative bacteria, and urethral catheterization ( $>3$ days)
were identified as independent risk factors of IFI in
patients with severe sepsis.
IFI is associated with excess risk for death in the hospi-
tal and greater consumption of medical resources.

\section{Competing interests}

The authors declare that they have no competing interests.

\section{Authors' contributions}

G-HX, X-MF, and B-LC contributed to the design of the study and drafted the manuscript. QF, X-MW, Y-HJ, J-LW, Q-LG, MNG, Q-PX, D-XW, S-LY, S-YY, Z-HD, and Y-BS obtained the data. G-HX, X-MF, B-LC, H-HW, and S-JW participated in data analysis and interpretation of the results.

\section{Acknowledgements}

The authors thank the following doctors and centers for their substantial help for this study: Drs Xinmin Wu and Dongxin Wang (Department of
Anesthesiology and Intensive Care Unit, the First Affiliated Hospital, School of Medicine, Peking Beijing University); Drs Shanglong Yao and Shiying Yuan (Department of Anesthesiology and Intensive Care Unit, Union hospital, Tongji School of Medicine School, Huazhong University of Science and Technology); Dr Zhaohui Du (Intensive Care Unit, Zhongnan Hosipital, School of Medicine, Wuhan University); Dr Qulian Guo (Department of Anesthesiology and Intensive Care Unit, Xiangya Hospital, Xiangya Medical College, Central South University); Dr Miaoning Gu (Department of Anesthesiology and Intensive Care Unit, South hospital, South Medical University [Wang Ruiting]); Dr Yunbo Sun (Department of Anesthesiology and Intensive Care Unit, the Affiliated Hospital of Qingdao University, School of Medicine, Qingdao University [Sun YunBo]); Dr Qiang Fang (Department of Anesthesiology, Intensive Care Unit, the First Affiliated Hospital, School of Medicine, Zhejiang University); Dr Qiuping Xu (Intensive Care Unit, Sir Run Run Shaw Hospital, School of Medicine, Zhejiang University); Dr Yuhong Jin (Intensive Care Unit, Lihuili hospital, School of Medicine, Ningbo University); and Dr Junlu Wang (Department of Anesthesiology, the First Affiliated Hospital, Wenzhou Medical College).

The participating university hospitals were as follows: the First Affiliated Hospital of Beijing University, Beijing; Union Hospital, Affiliated Hospital of Huazhong University of Science and Technology, Wuhan; Zhongnan hosipital, Affiliated Hospital of Wuhan University, Wuhan; Xiangya Hospital, Affiliated Hospital of Centre-south University, Changsha; South Hospital, Affiliated Hospital of South Medical University, Guangdong; the Affiliated Hospital of Qingdao University, Qingdao; the First Affiliated Hospital of Zhejiang University, Hangzhou; Sir Run Run Shaw Hospital, Affiliated Hospital of Zhejiang University, Hangzhou; Lihuili Hospital, Affiliated Hospital of Ningbo University, Ningbo; and the First Affiliated Hospital of Wenzhou Medical College, Wenzhou. This work was financially supported by National Natural Science Foundation of China (XMF, no. 30471662) and Program for New Century Excellent Talents in University (XMF, no. NCET-05-0522).

\section{References}

1. Eggimann P, Garbino J, Pittet D: Epidemiology of Candida species infections in critically ill non-immunosuppressed patients. Lancet Infect Dis 2003, 3:685-702.

2. Fisher-Hoch SP, Hutwagner L: Opportunistic candidiasis: an epidemic of the 1980s. Clin Infect Dis 1995, 21:897-904.

3. Fridkin SK, Jarvis WR: Epidemiology of nosocomial fungal infections. Clin Microbiol Rev 1996, 9:499-511.

4. Vincent JL, Sakr Y, Sprung CL, Ranieri VM, Reinhart K, Gerlach H, Moreno R, Carlet J, Le Gall JR, Payen D: Sepsis in European intensive care units: results of the SOAP study. Crit Care Med 2006, 34:344-353.

5. Brun-Buisson C, Doyon F, Carlet J, Dellamonica P, Gouin F, Lepoutre A, Mercier JC, Offenstadt G, Regnier B: Incidence, risk factors, and outcome of severe sepsis and septic shock in adults: a multicenter prospective study in intensive care units. JAMA 1995, 274:968-974.

6. Sands KE, Bates DW, Lanken PN, Graman PS, Hibberd PL, Kahn $\mathrm{KL}$, Parsonnet J, Panzer R, Orav EJ, Snydman DR, Black E, Schwartz JS, Moore R, Johnson BL Jr, Platt R, Academic Medical Center Consortium Sepsis Project Working Group: Epidemiology of sepsis syndrome in 8 academic medical centers. JAMA 1997, 278:234-240.

7. Wichmann MW, Inthorn D, Andress HJ, Schildberg FW: Incidence and mortality of severe sepsis in surgical intensive care patients: the influence of patient gender on disease process and outcome. Intensive Care Med 2000, 26:167-172.

8. Finfer S, Bellomo R, Lipman J, French C, Dobb G, Myburgh J: Adult-population incidence of severe sepsis in Australia and New Zealand intensive care units. Intensive Care Med 2004, 30:589-596.

9. Adrie C, Alberti C, Chaix-Couturier C, Azoulay E, De Lassence A, Cohen Y, Meshaka P, Cheval C, Thuong M, Troché G, Garrouste- 
Orgeas M, Timsit JF: Epidemiology and economic evaluation of severe sepsis in France: age, severity, infection site, and place of acquisition (community, hospital, or intensive care unit) as determinants of workload and cost. J Crit Care 2005, 20:46-58.

10. Silva E, Pedro Mde A, Sogayar AC, Mohovic T, Silva CL, Janiszewski M, Cal RG, de Sousa EF, Abe TP, de Andrade J, de Matos JD, Rezende E, Assuncão M, Avezum A, Rocha PC, de Matos GF, Bento AM, Corrêa AD, Vieira PC, Knobel E, Brazilian Sepsis Epidemiological Study: Brazilian Sepsis Epidemiological Study (BASES study). Crit Care 2004, 8:R251-R260.

11. Padkin A, Goldfrad C, Brady AR, Young D, Black N, Rowan K: Epidemiology of severe sepsis occurring in the first $24 \mathrm{~h}$ in intensive care units in England, Wales, and Northern Ireland. Crit Care Med 2003, 31:2332-2338.

12. Alberti C, Brun-Buisson C, Burchardi H, Martin C, Goodman S, Artigas A, Sicignano A, Palazzo M, Moreno R, Boulmé R, Lepage E, Le Gall R: Epidemiology of sepsis and infection in ICU patients from an international multicentre cohort study. Intensive Care Med 2002, 28:108-121.

13. Guidet B, Aegerter P, Gauzit R, Meshaka P, Dreyfuss D, CUB-Rea Study Group: Incidence and impact of organ dysfunctions associated with sepsis. Chest 2005, 127:942-951.

14. Martin GS, Mannino DM, Eaton S, Moss M: The epidemiology of sepsis in the United States from 1979 through 2000. N Engl J Med 2003, 348:1546-1554.

15. Raymond DP, Pelletier SJ, Crabtree TD, Gleason TG, Pruett TL, Sawyer RG: Impact of bloodstream infection on outcomes among infected surgical inpatients. Ann Surg 2001, 233:549-555.

16. Zaoutis TE, Argon J, Chu J, Berlin JA, Walsh TJ, Feudtner C: The epidemiology and attributable outcomes of candidemia in adults and children hospitalized in the United States: a propensity analysis. Clin Infect Dis 2005, 41:1232-1239.

17. Morgan J, Meltzer Ml, Plikaytis BD, Sofair AN, Huie-White S, Wilcox S, Harrison LH, Seaberg EC, Hajjeh RA, Teutsch SM: Excess mortality, hospital stay, and cost due to candidemia: a casecontrol study using data from population-based candidemia surveillance. Infect Control Hosp Epidemiol 2005, 26:540-547.

18. Annane $D$, Aegerter $P$, Jars-Guincestre MC Guidet B, CUB-Réa Network: Current epidemiology of septic shock: the CUB-Réa Network. Am J Crit Care Med 2003, 168:165-172.

19. Knaus WA, Draper EA, Wagner DP, Zimmerman JE: APACHE II: a severity of disease classification system. Crit Care Med 1985, 13:818-829.

20. Vincent JL, Moreno R, Takala J, Willatts S, De Mendonca A, Bruining $H$, Reinhart CK, Suter PM, Thijs LG: The SOFA (Sepsisrelated Organ Failure Assessment) score to describe organ dysfunction/failure. Intensive Care Med 1996, 22:707-710.

21. Ferreira FL, Bota DP, Bross A, Mélot C, Vincent JL: Serial evaluation of the SOFA score to predict outcome in critically ill patients. JAMA 2001, 286:1754-1758.

22. Girou E, Stephan F, Novara A, Safar M, Fagon JY: Risk factors and outcome of nosocomial infections: results of a matched case-control study of ICU patients. Am J Respir Crit Care Med 1998, 157:1151-1158.

23. Ascioglu $\mathrm{S}$, Rex JH, de Pauw B, Bennett JE, Bille J, Crokaert F, Denning DW, Donnelly JP, Edwards JE, Erjavec Z, Fiere D, Lortholary O, Maertens J, Meis JF, Patterson TF, Ritter J, Selleslag D, Shah PM, Stevens DA, Walsh TJ, Invasive Fungal Infections Cooperative Group of the European Organization for Research and Treatment of Cancer; Mycoses Study Group of the National Institute of Allergy and Infectious Diseases: Defining opportunistic invasive fungal infections in immunocompromised patients with cancer and hematopoietic stem cell transplants: an international consensus. Clin Infect Dis 2002, 34:7-14.

24. Vandewoude KH, Blot SI, Depuydt P, Benoit D, Temmerman W, Colardyn F, Vogelaers D: Clinical relevance of Aspergillus isolation from respiratory tract samples in critically ill patients. Crit Care 2006, 10:R31.

25. Levy MM, Fink MP, Marshall JC, Abraham E, Angus D, Cook D, Cohen J, Opal SM, Vincent JL, Ramsay G, SCCM/ESICM/ACCP/ ATS/SIS: 2001 SCCM/ESICM/ACCP/ATS/SIS International Sepsis Definitions Conference. Crit Care Med 2003, 31:1250-1256.
26. Volk HD: Immunodepression in the surgical patient and increased susceptibility to infection. Crit Care 2002, 6:279-281.

27. Rangel-Frausto MS, Martin MA, Saiman L, the NEMIS study group: High prevalence of Candida spp. on hands of healthcare workers in surgical and neonatal intensive care units: a multicenter study [abstract J106]. In Proceedings and abstracts of the 34th Interscience Conference on Antimicrobial Agents and Chemotherapy Orlando, Florida; 4 to 7 October 1994. Washington, DC: American Society for Microbiology; 1994.

28. Liu $X Y, X u Y$, Wen SK: Risk factors of iatrogenic fungal infection in intensive care unit. Chin J Nosocomiol 2005, 15:31-32.

29. Almirante B, Rodríguez D, Park BJ, Cuenca-Estrella M, Planes AM, Almela M, Mensa J, Sanchez F, Ayats J, Gimenez M, Saballs P, Fridkin SK, Morgan J, Rodriguez-Tudela JL, Warnock DW, Pahissa A, Barcelona Candidemia Project Study Group: Epidemiology and predictors of mortality in cases of Candida bloodstream infection: results from population-based surveillance, barceIona, Spain, from 2002 to 2003. J Clin Microbiol 2005, 43:1829-1235.

30. Cheng MF, Yu KW, Tang RB, Fan YH, Yang YL, Hsieh KS, Ho M, Lo $\mathrm{HJ}$ : Distribution and antifungal susceptibility of Candida species causing candidemia from 1996 to 1999. Diagn Microbiol Infect Dis 2004, 48:33-37.

31. Trick WE, Fridkin SK, Edwards JR, Hajjeh RA, Gaynes RP, National Nosocomial Infections Surveillance System Hospitals: Secular trend of hospital-acquired candidemia among intensive care unit patients in the United States during 1989-1999. Clin Infect Dis 2002, 35:627-630.

32. Blumberg HM, Jarvis WR, Soucie JM, Edwards JE, Patterson JE, Pfaller MA, Rangel-Frausto MS, Rinaldi MG, Saiman L, Wiblin RT, Wenzel RP, National Epidemiology of Mycoses Survey (NEMIS) Study Group: Risk factors for candidal bloodstream infections in surgical intensive care unit patients: the NEMIS prospective multicenter study. The National Epidemiology of Mycosis Survey. Clin Infect Dis 2001, 33:177-186.

33. Vincent $\mathrm{JL}$, Anaissie E, Bruining $\mathrm{H}$, Demajo W, el-Ebiary M, Haber J, Hiramatsu Y, Nitenberg G, Nystrom PO, Pittet D, Rogers T, Sandven P, Sganga G, Schaller MD, Solomkin J: Epidemiology, diagnosis and treatment of systemic Candida infection in surgical patients under intensive care. Intensive Care Med 1998 24:206-216

34. Diekema DJ, Messer SA, Brueggemann AB, Coffman SL, Doern GV, Herwaldt LA, Pfaller MA: Epidemiology of candidemia: 3year results from the emerging infections and the epidemiology of lowa organisms study. J Clin Microbiol 2002 40:1298-1302.

35. Nolla-Salas J, Sitges-Serra A, León-Gil C, Martínez-González J, León-Regidor MA, Ibáñez-Lucía $P$, Torres-Rodríguez JM: Candidemia in non-neutropenic critically ill patients: analysis of prognostic factors and assessment of systemic antifungal therapy. Intens Care Med 1997, 23:23-30.

36. Morgan J, Meltzer MI, Plikaytis BD, Sofair AN, Huie-White S, Wilcox S, Harrison LH, Seaberg EC, Hajieh RA, Teutsch SM: Excess mortality, hospital stay, and cost due to candidemia: a casecontrol study using data from population-based candidemia surveillance. Infect Control Hosp Epidemiol 2005, 26:540-547.

37. Zaoutis TE, Argon J, Chu J, Berlin JA, Walsh TJ, Feudtner C: The epidemiology and attributable outcomes of candidemia in adults and children hospitalized in the United States: a propensity analysis. Clin Infect Dis 2005, 41:1232-1239.

38. Digiovine $B$, Chenoweth $C$, Watts $C$, Higgins $M$ : The attributable mortality and costs of primary nosocomial bloodstream infections in the intensive care unit. Am J Respir Crit Care Me 1999, 160:976-981.

39. Blot SI, Vandewoude $\mathrm{KH}$, Hoste EA, Colardyn FA: Effects of nosocomial candidemia on outcomes of critically ill patients. Am J Med 2002, 113:480-485. 